\title{
O USO DO BALANCED SCORECARD COMO INSTRUMENTO DE MEDIÇÃO PARA COMPARAR OS MODELOS DE EXCELÊNCIA EM GESTÃO
}

\section{RESUMO}

Este trabalho tem como objetivo fazer uma análise comparativa dos modelos de excelência em gestão European Foundation Quality Management (EFQM), Baldridge Performance Excellence Program (BPEP) e Modelo de Excelência da Gestão (MEG), utilizando como instrumento de medição as perspectivas do Balanced Scorecard (BSC). A metodologia utilizada foi a análise de conteúdo, onde se buscou analisar a pontuação dos critérios e dos subcritérios de cada um dos modelos, relacionando-os com as perspectivas do BSC. Os resultados demonstram os pontos similares entre os três modelos avaliados (EFQM, BPEP e MEG), bem como um relacionamento entre cada subcritério e sua respectiva pontuação com as perspectivas do BSC, sendo possível quantificar percentualmente quanto cada modelo contribui para cada perspectiva do BSC.

Palavras chave: BSC; EFQM; Malcolm Baldridge; MEG.

\section{USING THE BALANCED SCORECARD AS A MEASUREMENT TOOL FOR COMPARING MODELS OF EXCELLENCE IN MANAGEMENT}

\begin{abstract}
This work aims to make a comparative analysis of management excellence models European Foundation Quality Management (EFQM), Baldridge Performance Excellence Program (BPEP) and Modelo de Excelência da Gestão (MEG), using BSC's perspectives as a measuring tool. The methodology used was content analysis, where it sought to analyze the score of the criteria and sub-criteria for each of the models and their relationships with BSC's perspectives. The results show similar points between the three models tested (EFQM, BPEP and MEG) as well as a relationship between each sub-criteria and their respective scores with the BSC perspectives, making it possible to quantify how each model contributed for each perspective of the BSC.
\end{abstract}

Keywords: BSC; EFQM; Malcolm Baldridge; MEG. 
El Uso del Cuadro de Mando Integral como Instrumento de Medición para Comparar los Modelos de Excelencia en Gestión

\section{EL USO DEL CUADRO DE MANDO INTEGRAL COMO INSTRUMENTO DE MEDICIÓN PARA COMPARAR LOS MODELOS DE EXCELENCIA EN GESTIÓN}

\section{RESUMEN}

Este trabajo tiene como objetivo hacer un análisis comparativo de los modelos de excelencia en gestión European Foundation Quality Management (EFQM), Baldridge Performance Excellence Program (BPEP) y Modelo de Excelência da Gestão (MEG), utilizando como instrumento de medición las perspectivas del Cuadro de Mando Integral (CMI). La metodología utilizada fue el análisis de contenido, donde se buscó analizar la puntuación de los criterios y subcriterios de cada uno de los modelos y sus relaciones con las perspectivas del CMI. Los resultados enseñan puntos similares entre los tres modelos evaluados (EFQM, BPEP y MEG), así como una relación entre cada subcriterio y sus respectivas puntuaciones a las perspectivas del CMI, siendo posible cuantificar una contribución porcentual de cada modelo para cada perspectiva del CMI.

Palabras clave: Cuadro de Mando Integral; EFQM; Malcolm Baldridge; MEG.

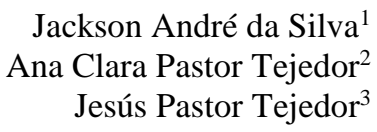

\footnotetext{
${ }^{1}$ Doutorando da Faculdad de Economía y Empresa na Universidad de Zaragoza, UNIZAR, Espanha. Coordenador do Núcleo de Planejamento do Sebrae/SC. Brasil. E-mail: jackson@sc.sebrae.com.br

${ }^{2}$ Profesora Titular del Dpto. de Dirección y Organización de Empresas da Escuela de Ingeniería y Arquitectura de la Universidad de Zaragoza. Brasil. E-mail: acpastor@unizar.es

${ }^{3}$ Profesor Contratado Doctor Dpto. de Dirección y Organización de Empresas Escuela de Ingeniería y Arquitectura de la Universidad de Zaragoza. Brasil. E-mail: jpastej@unizar.es
} 


\section{INTRODUCCIÓN}

El ránking de las empresas latinoamericanas en proceso de globalización en 2013, presentó un total de 80 empresas, siendo 16 nuevas, comparadas al ranking del año de 2012 (América Economía, 2013). Esto da cuenta de cómo el fenómeno multilatino se expande. Entre los países con mayor número de multinacionales en el ranking, destacan Brasil con 25 empresas, seguido por Chile con 19, sin contar la empresa Latan, que surgió de la fusión entre la brasileña TAM y la chilena LAN, y el México con 18 empresas multinacionales. El éxito de las empresas brasileñas, tuvo origen con la expansión internacional, empezada en los años 90 (Correa, Paiva \& Primo, 2010), teniendo como destino los EE.UU., Argentina, Chile, Colombia y Uruguay (FDC, 2013).

De acuerdo Rovai, Campanário \& Costa (2011), las multinacionales son las empresas que más incorporan o desarrollan nuevas tecnologías, sistemas y procesos productivos, las que tienen acceso a líneas de crédito con menores costes y las que cualifican mejor su gestión, buscando así obtener diversas certificaciones internacionales de calidad. Sin embargo, dependiendo de donde estas empresas actúan, se depararan con una gran diversidad de modelos de evaluación, también conocidos como "modelos de excelencia en gestión".

Los atributos de calidad deben ser empleados en las estrategias de las organizaciones, buscando garantizar objetivos claros y recursos financieros compatibles con sus necesidades y con las expectativas de las partes interesadas, contribuyendo al desarrollo de los procesos internos y fomentando el aprendizaje y el crecimiento a largo plazo.

De esta manera, un análisis que puede ser realizado es la integración de los modelos de excelencia en gestión con el cuadro de mando integral (CMI), buscando evaluar como los modelos contribuyen para el alcance de visión y de las estrategias empresariales.

La integración propuesta parte del uso de las cuatro perspectivas del CMI como base de comparación de los modelos de calidad y de gestión de la excelencia empresarial. Así, es posible identificar que enfoque o perspectiva pondera más cada modelo para conseguir la excelencia.

Según Kaplan \& Norton (1992) el CMI busca esclarecer, obtener el consenso y el enfoque en las estrategias, haciendo que sea más sencillo comunicarlas. Sin embargo, el principal beneficio del uso del CMI es alcanzado cuando él deja de ser un simple sistema de evaluación basado en indicadores (control) y se torna un sistema integrado de gestión estratégica, que tiene como enfoque principal la visión y la estrategia.
Autores como Miguel (2001), Vilas Boas \& Costa (2011), Vasconcellos \& Lucas (2012) han comparado el modelo de excelencia en gestión brasileño (MEG) y el CMI, pero sin intentar hacerlo relacionando los subcriterios de los modelos de excelencia con las perspectivas del CMI. Otros trabajos como los de Pastor y otros (2008), Dror (2008), Akhavan y otros (2013) han buscado integrar los Modelos EFQM y Malcolm Baldridge con el CMI, pero también usando un enfoque diferente del utilizado en este trabajo, que tiene el mismo enfoque del trabajado elaborado por Pastor y otros (2013), pero que intenta ir más allá, incorporando en el análisis el modelo de excelencia en gestión brasileño (MEG).

Así, este artículo busca hacer un análisis comparativo de los modelos de excelencia en gestión EFQM, Malcolm Baldridge y MEG, utilizando como instrumento de medición las perspectivas del CMI. Además, destacase que es importante que los procesos de gestión conduzcan a la consecución de los objetivos estratégicos, lo que refuerza la importancia de realizar un análisis conjunto de los modelos de excelencia en gestión y el cuadro de mando integral (CMI), observando las interacciones entre ellos.

En el apartado 2, son presentados los conceptos sobre el CMI y los tres modelos de excelencia en gestión utilizados en el presente trabajo. En el apartado 3, está presentada la metodología y en el apartado 4 los resultados encontrados, finalizado con el apartado 5, donde es posible observar las conclusiones y recomendaciones de trabajos futuros.

\section{FUNDAMENTACIÓN TEÓRICA}

Este apartado empieza con la descripción del CMI y de sus perspectivas. Sigue con los conceptos de los modelos de excelencia en gestión EFQM, Malcolm Baldridge y MEG, sus objetivos originales, relaciones entre los criterios, subcriterios y las puntuaciones atribuidas en sus sistemas de evaluación.

\subsection{Cuadro de Mando Integral}

El CMI es una herramienta de trabajo que utiliza un conjunto de medidas para garantizar que la alta dirección tenga una visión rápida, pero comprensiva de su negocio. Parte de la misión, la visión y la estrategia, desmembrándolos en objetivos que son supervisados por medio del establecimiento de metas mensurables, conocidas como indicadores de desempeño. También crea un mapa estratégico, utilizando un lenguaje simplificado que facilita la comunicación de la visión y de las estrategias de la empresa (Kaplan \& Norton, 1992).

El modelo está estructurado en cuatro perspectivas que reflejan la misión, la visión y las 
estrategias en objetivos medibles: financiera, clientes, procesos internos y aprendizaje y crecimiento, de acuerdo con la Figura 1.

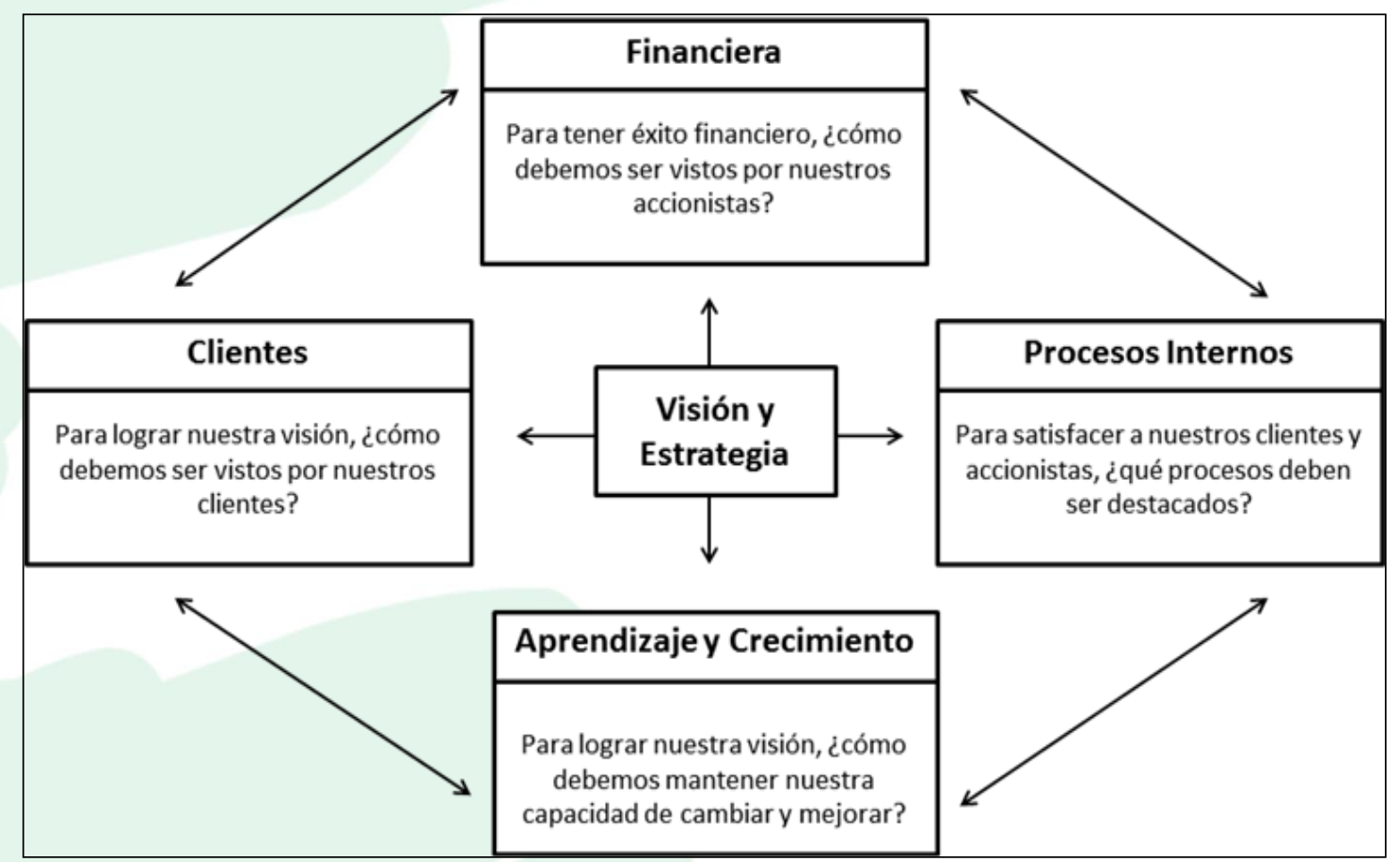

Figura 1 - Perspectivas del modelo Balance Scored Card. (Cuadro de Mando Integral, CMI) Fuente: Kaplan y Norton (1996a).

En la parte superior del modelo está la perspectiva financiera, que de acuerdo con Kaplan y Norton (1992, 1996a), indica si la ejecución de los indicadores de las otras perspectivas están contribuyendo a la mejora de los indicadores financieros, en una relación de abajo hasta arriba. Es decir, los indicadores de esta perspectiva indican si la estrategia de la empresa, su implementación y ejecución están contribuyendo a la mejora de los resultados financieros. Los objetivos financieros buscan la supervivencia, el éxito y la prosperidad de la empresa. Cualquier medida seleccionada debe hacer parte de una cadena de causa y efecto, que culminan en una mejora de los resultados financieros.

La perspectiva de los clientes suele contener objetivos e indicadores que representan la segmentación del mercado en los que la compañía opera o pretende operar. Medidas comúnmente usadas incluyen la satisfacción y la retención de clientes, la rentabilidad por segmentos y el aumento de la cuota de mercado (Kaplan \& Norton, 1992, 1996a).

Kaplan y Norton (1992, 1996a) desarrollaron la perspectiva de procesos internos para permitir a los directivos a identificar los procesos críticos donde la empresa debe tener la excelencia. Por lo tanto, los indicadores de esta perspectiva tienen como objetivo atraer y retener a los clientes y a satisfacer las expectativas de los accionistas en relación a los rendimientos financieros.

La cuarta perspectiva es la de aprendizaje y crecimiento, que se ocupa de la infraestructura y la innovación necesaria para desarrollar la empresa para lograr el crecimiento esperado y la mejora a largo plazo. Según Kaplan y Norton (1996b), es poco probable que las empresas sean capaces de alcanzar sus objetivos a largo plazo para las perspectivas de los clientes y de los procesos internos utilizando sólo la tecnología disponible hoy en día. Por lo tanto, las empresas tienen que invertir en el desarrollo de sus empleados, en los sistemas de información y en los procedimientos organizacionales.

El CMI utiliza indicadores internos y externos (financieros y no financieros) y establece una relación de causa-efecto entre los objetivos estratégicos definidos para las cuatro perspectivas, de modo que los objetivos de la perspectiva de aprendizaje y crecimiento, cuando alcanzados, producen mejoras en los procesos internos, que dejan los clientes más satisfechos y leales a la empresa. Al final, las expectativas de las partes interesadas son atendidas con las mejoras en los resultados financieros, ocurridas a 
El Uso del Cuadro de Mando Integral como Instrumento de Medición para Comparar los Modelos de Excelencia en Gestión

partir de los resultados obtenidos en las perspectivas de aprendizaje y crecimiento, procesos internos y clientes.

La construcción del CMI permite a las empresas asociar su presupuesto con los objetivos estratégicos y comunicar su estrategia de una manera simplificada. También les permite desarrollar un conjunto de mapas estratégicos, los cuales se pueden construir por unidad de negocio, lo que facilita la implementación y seguimiento de las estrategias, objetivos y metas.

\subsection{Modelo European Foundation Quality Management}

Creado en 1991 para mejorar la posición competitiva de las empresas europeas en el mercado global, el EFQM ha sido utilizado por más de 30.000 organizaciones, convirtiéndose en la herramienta de calidad más popular en Europa (EFQM, 2013).

El EFQM tiene nueve criterios que están divididos en dos categorías: los "medios" que comprenden los agentes facilitadores (actividades principales) y se representan en el lado izquierdo, y que necesitan ser mejorados para que los "resultados", agrupados en el lado derecho, sean logrados (Figura 2).

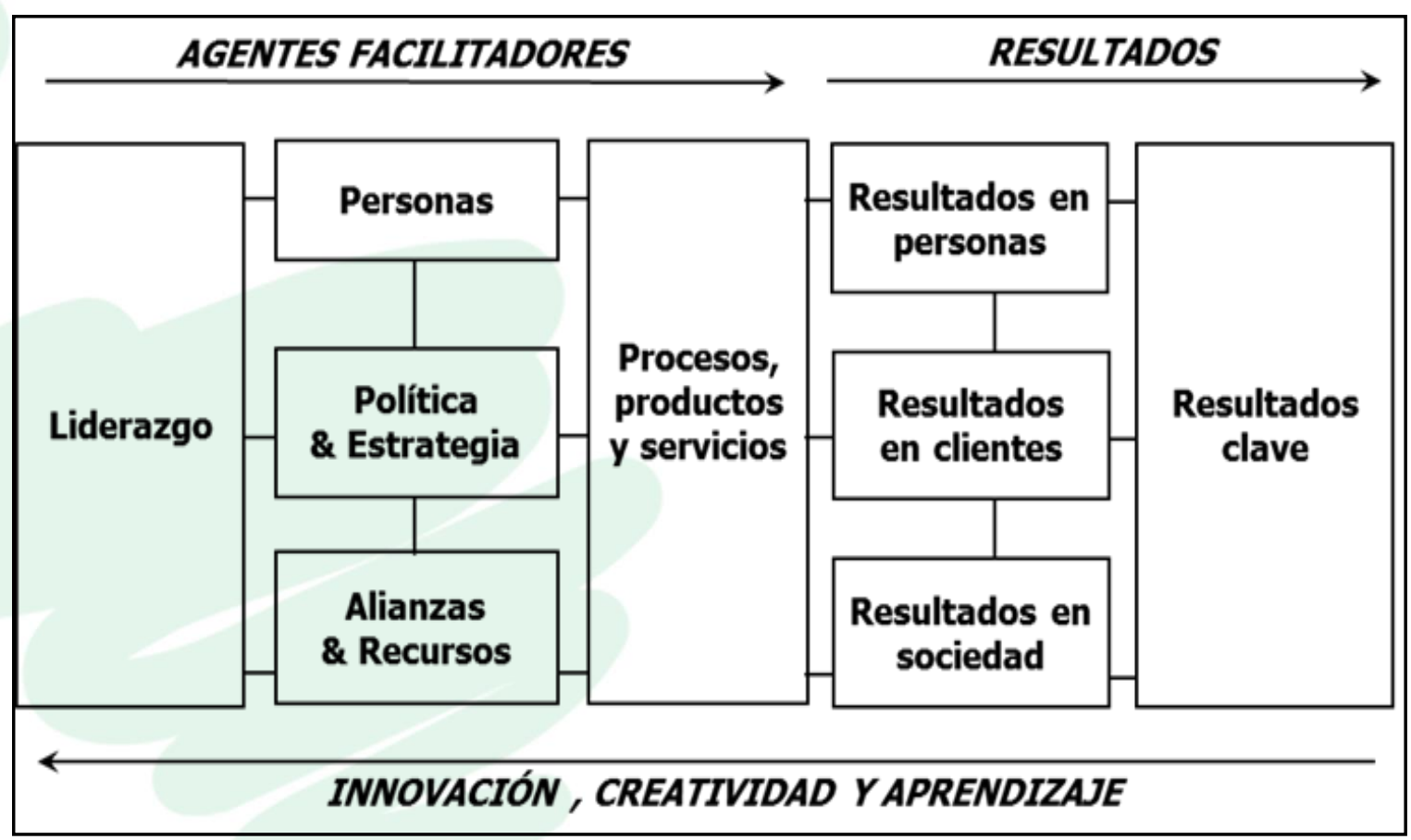

Figura 2 - Modelo EFQM.

Fuente: EFQM (2013).

La puntuación máxima que una empresa puede lograr con la aplicación del EFQM es de 1.000 puntos. La evaluación se hace con base en los nueve criterio, siguiendo lo descrito en el Cuadro 1, tomado del documento Introducing the EFQM Excellenge Model 2010 (EFQM, 2013).

\begin{tabular}{|l|c|}
\hline \multicolumn{1}{|c|}{ CRITERIOS DE EVALUACIÓN } & PUNTUACIÓN \\
\hline 1. Liderazgo & 100 \\
\hline 2. Política \& Estrategia & 100 \\
\hline 3. Personas & 100 \\
\hline 4. Alianzas \& Recursos & 100 \\
\hline 5. Procesos, Productos y Servicios & 100 \\
\hline 6. Resultados en Clientes & 150 \\
\hline 7. Resultados en Personas & 100 \\
\hline 8. Resultados en Sociedad & 100 \\
\hline 9. Resultados Clave & 150 \\
\hline Puntuación Total & $\mathbf{1 . 0 0 0}$ \\
\hline
\end{tabular}

Cuadro 1 - Criterios de Evaluación del Modelo EFQM. Fuente: EFQM (2010). 
El Uso del Cuadro de Mando Integral como Instrumento de Medición para Comparar los Modelos de Excelencia en Gestión

\subsection{Modelo Baldridge Performance Excellence Program}

Actualmente conocido como Baldridge Performance Excellence Program, el Malcolml Baldridge National Quality Award fue creado en 1987, un año antes del surgimiento de la Foundation for the Malcolm Baldridge National Quality Award, que es la responsable de la promoción y gestión de la entrega del premio (NIST, 2013).

En el centro modelo se encuentran seis criterios (Figura 3), que definen los procesos y los resultados deseados. Liderazgo, estrategia y enfoque en los clientes representan el trípode de liderazgo, donde los líderes deben enfatizar la atención a las estrategias y a los clientes. Personas, gestión de procesos y resultados representan el trípode del resultado, donde el trabajo de las personas y la organización de los procesos clave conducen al desempeño global. Finalmente, la medición, el análisis y la gestión del conocimiento son empleadas en la búsqueda de mejorar el rendimiento y la competitividad, haciendo con que la gestión de la organización sea más efectiva (NIST, 2013).

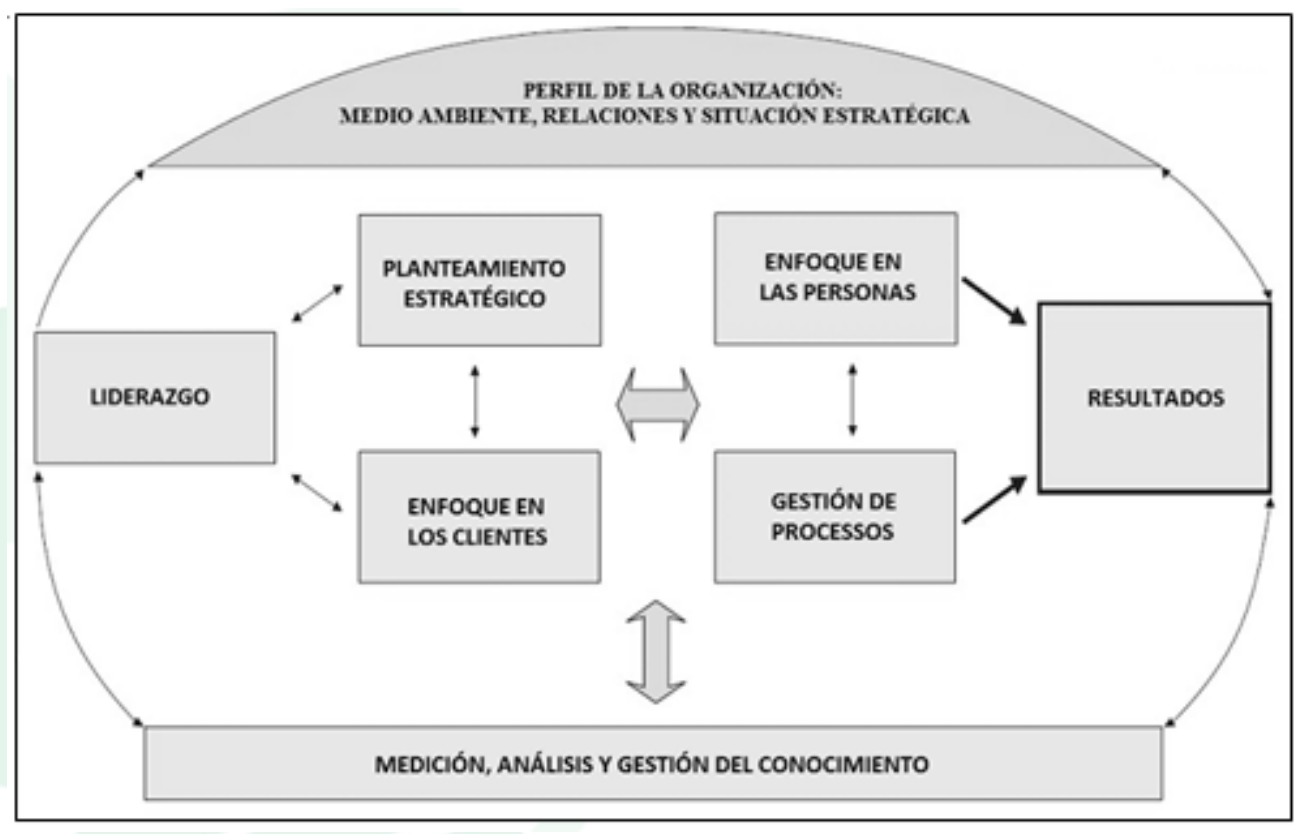

Figura 3 - Modelo BPEP.

Fuente: NIST (2013).

El sistema de puntuación también sigue la escala de 1.000 puntos, tales como el EFQM, y es obtenido mediante el análisis de siete criterios. El
Cuadro 2 enseña los criterios de evaluación y sus respectivas puntuaciones.

\begin{tabular}{|l|c|}
\hline \multicolumn{1}{|c|}{ CRITERIOS DE EVALUACIÓN } & PUNTUACIÓN \\
\hline 1. Liderazgo & 120 \\
\hline 2. Planteamiento Estratégico & 85 \\
\hline 3. Enfoque en los Clientes & 85 \\
\hline 4. Medición, Análisis y Gestión del Conocimiento & 90 \\
\hline 5. Enfoque en las Personas & 85 \\
\hline 6. Gestión Operacional & 85 \\
\hline 7. Resultados & 450 \\
\hline Puntuación Total & $\mathbf{1 . 0 0 0}$ \\
\hline
\end{tabular}

Cuadro 2 - Criterios de evaluación del modelo BPEP. Fuente: NIST (2013). 
El Uso del Cuadro de Mando Integral como Instrumento de Medición para Comparar los Modelos de Excelencia en Gestión

\subsection{MODELO DE EXCELÊNCIA DA GESTÃo}

El MEG fue creado en 1991 por la Fundación Nacional de la Calidad (FNQ), que surgió en el mismo año con la misión de "difundir los fundamentos de la excelencia en la gestión para aumentar la competitividad de las organizaciones y del Brasil" (FNQ, 2009, p. 8).
Es compuesto por ocho criterios que juntos simbolizan la interacción de la organización con el ambiente externo. Considera que la organización es un sistema adaptativo y orgánico, que interfiere y sufre interferencia del ambiente. Los componentes del modelo, rodeados de información y conocimiento, se relacionan entre sí para fomentar la generación de resultados (FNQ, 2009).

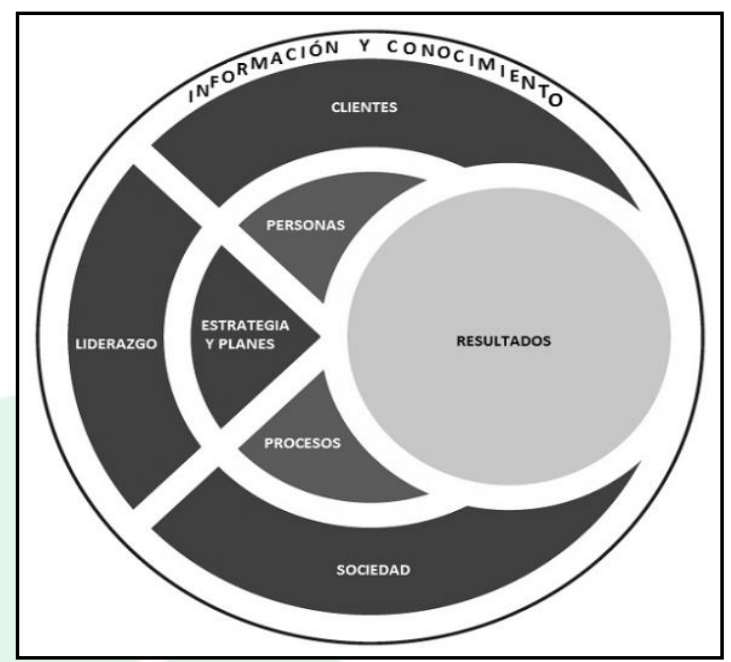

Figura 4 - Modelo MEG.

Fuente: FNQ (2009).

El sistema de puntuación se basa en la evaluación de cada uno de los ocho criterios del MEG, con la puntuación máxima obtenible de 1.000 puntos, así como la de los modelos EFQM y BPEP. Los criterios de evaluación y sus respectivas puntuaciones están descritos en el Cuadro 3.

\begin{tabular}{|l|c|}
\hline \multicolumn{1}{|c|}{ CRITERIOS DE EVALUACIÓN } & PUNTUACIÓN \\
\hline 1. Liderazgo & 110 \\
\hline 2. Estrategias y Planes & 60 \\
\hline 3. Clientes & 60 \\
\hline 4. Sociedad & 60 \\
\hline 5. Información y Conocimiento & 60 \\
\hline 6. Personas & 90 \\
\hline 7. Procesos & 110 \\
\hline 8. Resultados & 450 \\
\hline Puntuación Total & $\mathbf{1 . 0 0 0}$ \\
\hline
\end{tabular}

Cuadro 3 - Criterios de evaluación del modelo MEG. Fuente: FNQ (2009).

Finalizada la fundamentación teórica sobre el CMI y sobre los tres modelos de excelencia en gestión utilizados en este estudio, será descrita en la sesión siguiente la metodología científica aplicada al presente trabajo.

\section{METODOLOGÍA}

El presente trabajo se caracteriza como una investigación cualitativa, ya que busca analizar comparativamente los modelos de excelencia en gestión EFQM, Malcolm Baldridge y MEG, teniendo como base las perspectivas del CMI. Según Godoy (1995), la investigación cualitativa incluye datos 
descriptivos e interactivos, buscando comprender los fenómenos a partir de las perspectivas de las personas participantes en cada situación estudiada. Para él, este tipo de investigación está centrada en una descripción detallada de una situación a ser estudiada.

En cuanto a sus objetivos, esta investigación puede ser clasificada como descriptiva, porque analiza cuanto contribuye cada modelo de excelencia en gestión para conseguir los objetivos estratégicos de cada perspectiva del CMI. Estudios de naturaleza descriptiva proponen investigar las características de un fenómeno como tal (Richardson, 1999), sin tener la intención de cambiarlos (Marconi y Lakatos, 2009).
La primera etapa realizada fue la de organización del material para la conducción del análisis de contenido, que es un conjunto de técnicas con el objetivo de obtener, por medio de procedimientos sistemáticos y objetivos, una descripción del contenido del mensaje para que se pueda hacer inferencias y deducciones lógicas sobre él (Bardin, 2011). De acuerdo con Bardin (2011), la utilización del análisis de contenido establece tres fases fundamentales y consecutivas, conforme descrito en la Figura 5.

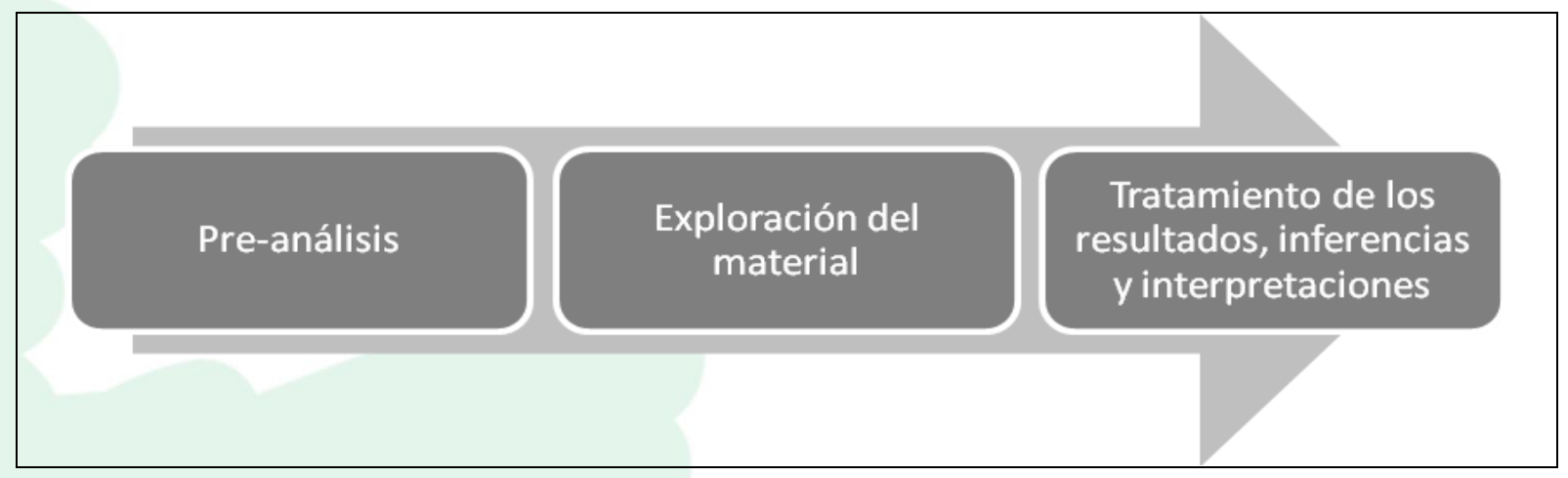

Figura 5 - Tres fases del análisis de contenido.

Fuente: Adaptada de Bardin (2011).

En la primera fase, llamada pre-análisis, fue establecido la organización del trabajo, la definición de los procedimientos y un primer contacto con los documentos, que permitió la selección de los documentos más adecuados. Aquí se obedeció a las reglas sugeridas por Bardin (2011) de exhaustividad (nada fue omitido), representatividad (la muestra seleccionada representó el universo de los modelos elegidos), homogeneidad (los datos se referían a los modelos de excelencia y al CMI, y fueron colectados con el uso de las mismas técnicas y por las mismas personas), pertinencia (los documentos seleccionados tratan el tema objeto de investigación) y exclusividad (cada subcriterio fue asignado solamente a una categoría/perspectiva del CMI).

Para la exploración del material se procedió con la elección de las unidades de codificación y definición de las categorías de análisis. En esta etapa se definió que las perspectivas del CMI serían utilizadas como categorías, permitiendo así una comparación entre los tres modelos seleccionados. Esta opción permitió realizar los procedimientos de codificación (selección de la unidad de medida), clasificación (agrupar por el sentido de las palabras) y categorización (unir informaciones para ordenarlos y correlacionarlos). En esta fase ha sido posible identificar las características y similitudes entre los modelos evaluados, buscando una forma de transformar los datos, en conocimiento. Los subcriterios de cada modelo fueron asignados de acuerdo con las perspectivas del CMI.

La tercera y última fase consistió en el tratamiento de los resultados, inferencias e interpretaciones, donde se buscó condensar y destacar las informaciones en un análisis reflexivo y crítico (Bardin, 2011). Al final de esta fase, fueron elaboradas tablas de equivalencias donde se asignaron las puntuaciones de los criterios y subcriterios de cada modelo de excelencia a las perspectivas del CMI, basándose en los conceptos descritos para cada perspectiva. A pesar de que los autores trabajan en la enseñanza y en evaluación de los modelos de excelencia en gestión, la etapa de asignación de las puntuaciones sería la principal limitación del trabajo, pues su validación ocurrió por medio de la búsqueda del consenso entre los autores.

Por fin, los resultados obtenidos con la comparación entre los modelos y el CMI fueron analizados, calculando los pesos atribuidos por los modelos a cada una de las perspectivas del CMI, conforme será descrito en el próximo apartado.

\section{RESULTADOS}

El análisis ha empezado con la comparación entre los objetivos y la estructura de los tres modelos de excelencia elegidos para la realización de este trabajo. Todos los modelos fueron elaborados para 
El Uso del Cuadro de Mando Integral como Instrumento de Medición para Comparar los Modelos de Excelencia en Gestión

mejorar la competitividad de las empresas de sus respectivos países/regiones y presentan una naturaleza no prescriptiva, o sea, no ofrecen un conjunto de herramientas de control. Trabajan con acciones de mejoría continua que pueden tornar las empresas mejores y más competitivas, buscando una evaluación del ciclo de madurez de gestión organizacional en que las empresas se encuentran.

Ofrecen un sistema de puntuación y el máximo que puede ser obtenido por una empresa en los tres modelos es 1.000 puntos, divididos por criterios y subcriterios. Aquí empieza la primera diferencia entre los modelos, ya que el EFQM es compuesto por nueve criterios, mientras que el BPEP tiene siete y el MEG ocho. Como hay divergencia entre la cantidad de criterios y subcriterios, se cree que la comparación con el CMI presentará diferencias interesantes sobre cuanto los modelos contribuyen con cada perspectiva del CMI.

A pesar de presentaren diferencias entre la cantidad total de criterios, hay algunas concordancias entre ellos, siendo que los criterios liderazgo, estrategia y planes, clientes, personas, procesos y resultados están presentes en los tres modelos. Sin embargo, los modelos BPEP y MEG enseñan una preocupación con la gestión de información y del conocimiento, mientras el EFQM presenta un interese en las alianzas y recursos.

En una visión general de la puntuación también es posible observar que el EFQM posee un equilibrio entre los criterios de los agentes facilitadores y de resultado, ya que cada grupo es evaluado en 500 puntos. Ya los modelos BPEP y MEG ofrecen una puntuación más pequeña para los criterios de resultados, donde en los dos modelos es posible obtener un máximo de 450 puntos con ellos, mientras los otros criterios poseen un peso de 550 puntos.

La segunda parte del análisis inicia con la asignación de los criterios y subcriterios de los tres modelos de excelencia en gestión con las perspectivas del CMI. El primer modelo analizado fue el EFQM, que posee nueve criterios y 32 subcriterios (Tabla 1).

Tabla 1 - Comparación del EFQM con el CMI.

\begin{tabular}{|c|c|c|c|}
\hline CRITERIOS & P. & SUBCRITERIOS & CMI \\
\hline \multirow{5}{*}{$\begin{array}{l}\text { 1.LIDERAZGO } \\
\text { 100 PUNTOS }\end{array}$} & 20 & $\begin{array}{l}\text { 1a. Los líderes desarrollan la misión, visión, valores y principios éticos y actúan } \\
\text { como modelo de referencia. }\end{array}$ & $\mathrm{V}$ \\
\hline & 20 & $\begin{array}{l}\text { 1b. Los líderes definen, supervisan, revisan e impulsan tanto la mejora del sistema de } \\
\text { gestión de la organización como su rendimiento }\end{array}$ & $\mathrm{P}$ \\
\hline & 20 & 1c. Los líderes se implican con los grupos de interés externos. & $\mathrm{C}$ \\
\hline & 20 & $\begin{array}{l}\text { 1d. Los líderes refuerzan una cultura de excelencia entre las personas de la } \\
\text { organización. }\end{array}$ & A \\
\hline & 20 & $\begin{array}{l}\text { 1e. Los líderes se aseguran de que la organización sea flexible y gestionan el cambio } \\
\text { de manera eficaz. }\end{array}$ & A \\
\hline \multirow{4}{*}{$\begin{array}{c}\text { 2. POLÍTICA Y } \\
\text { ESTRATEGIA } \\
100 \text { PUNTOS }\end{array}$} & 25 & $\begin{array}{l}\text { 2a. La estrategia se basa en comprender las necesidades y expectativas de los grupos } \\
\text { de interés y del entorno externo }\end{array}$ & $\mathrm{V}$ \\
\hline & 25 & $\begin{array}{l}\text { 2b. La estrategia se basa en comprender el rendimiento de la organización y sus } \\
\text { capacidades. }\end{array}$ & $\mathrm{V}$ \\
\hline & 25 & 2c. La estrategia y sus políticas de apoyo se desarrollan, revisan y actualizan. & $\mathrm{V}$ \\
\hline & 25 & 2d. La estrategia y sus políticas de apoyo se comunican, implantan y supervisan & $\mathrm{V}$ \\
\hline \multirow{5}{*}{$\begin{array}{l}\text { 3. PERSONAS } \\
\text { 100 PUNTOS }\end{array}$} & 20 & 3a. Los planes de gestión de personas apoyan la estrategia de la organización. & A \\
\hline & 20 & $\begin{array}{l}\text { 3b. Se desarrolla el conocimiento y las capacidades de las personas de la } \\
\text { organización }\end{array}$ & A \\
\hline & 20 & $\begin{array}{l}\text { 3c. Las personas están alineadas con las necesidades de la organización, implicadas y } \\
\text { asumen su responsabilidad. }\end{array}$ & A \\
\hline & 20 & 3d. Las personas se comunican eficazmente en toda la organización. & A \\
\hline & 20 & 3e. Recompensa, reconocimiento y atención a las personas de la organización. & A \\
\hline \multirow{5}{*}{$\begin{array}{l}\text { 4. ALIANZAS Y } \\
\text { RECURSOS } \\
100 \text { PUNTOS }\end{array}$} & 20 & 4a. Gestión de alianzas y proveedores para obtener un beneficio sostenible. & $\mathrm{P}$ \\
\hline & 20 & 4b. Gestión de los recursos económico-financieros para asegurar un éxito sostenible & $\mathrm{F}$ \\
\hline & 20 & 4c. Gestión sostenible de edificios, equipos, materiales y recursos naturales. & A \\
\hline & 20 & 4d. Gestión de la tecnología para hacer realidad la estrategia. & A \\
\hline & 20 & $\begin{array}{l}\text { 4e. Gestión de la información y del conocimiento para apoyar una eficaz toma de } \\
\text { decisiones y construir las capacidades de la organización }\end{array}$ & A \\
\hline \multirow{3}{*}{$\begin{array}{l}\text { 5. PROCESOS, } \\
\text { PRODUTOS Y } \\
\text { SERVICIOS } \\
100 \text { PUNTOS }\end{array}$} & 20 & $\begin{array}{l}\text { 5a. Los procesos se diseñan y gestionan a fin de optimizar el valor para los grupos de } \\
\text { interés. }\end{array}$ & $\mathrm{P}$ \\
\hline & 20 & 5b. Los productos y servicios se desarrollan para dar valor óptimo a los clientes. & $\mathrm{P}$ \\
\hline & 20 & 5c. Los productos y servicios se promocionan y ponen en el mercado eficazmente. & $\mathrm{P}$ \\
\hline
\end{tabular}


El Uso del Cuadro de Mando Integral como Instrumento de Medición para Comparar los Modelos de Excelencia en Gestión

\begin{tabular}{|c|c|l|c|}
\hline & 20 & 5d. Los productos y servicios se producen, distribuyen y gestionan. & P \\
\cline { 2 - 5 } & 20 & 5e. Las relaciones con los clientes se gestionan y mejoran. & $\mathrm{C}$ \\
\hline \multirow{2}{*}{$\begin{array}{c}\text { 6. RESULTADOS EN } \\
\text { CLIENTES } \\
\text { 150 PUNTOS }\end{array}$} & 75 & 6a. Percepciones. & $\mathrm{C}$ \\
\cline { 2 - 5 } $\begin{array}{c}\text { 7. RESULTADOS EN } \\
\text { PERSONAS } \\
\text { 100 PUNTOS }\end{array}$ & 75 & 6b. Indicadores de rendimiento. & $\mathrm{C}$ \\
\cline { 2 - 5 } $\begin{array}{c}\text { 8. RESULTADOS EN } \\
\begin{array}{c}\text { SOCIEDAD } \\
\text { 100 PUNTOS }\end{array}\end{array}$ & 50 & 7a. Percepciones. & $\mathrm{A}$ \\
\hline $\begin{array}{c}\text { 9. RESULTADOS } \\
\text { CLAVE }\end{array}$ & 75 & 8a. Percepciones. & $\mathrm{A}$ \\
\cline { 2 - 5 } 150 PUNTOS & 75 & 9b. Indicadores de rendimiento & $\mathrm{C}$ \\
\hline TOTAL & $\mathbf{1 . 0 0 0}$ & & $\mathrm{C}$ \\
\hline
\end{tabular}

Notas: V=Visión y estrategia; F=Financiera; $\mathrm{C}=$ Cliente; $\mathrm{P}=$ Procesos; $\mathrm{A}=$ Aprendizaje y Crecimiento.

Fuente: Elaboración propia.

Las perspectivas pueden ser separadas en dos grupos. El primer grupo es el más relevante. Contiene las perspectivas de aprendizaje y crecimiento y de clientes, ya que ellas tienen un peso mayor en este modelo (300 y 290 puntos, respectivamente). El segundo grupo está formado por las perspectivas financiera y procesos internos y por la visión y estrategia, con una puntuación menos relevante, ya que obtuvieron 170 y 120 puntos, respectivamente.

El segundo modelo analizado fue el BPEP que tiene siete criterios y 17 subcriterios. Los resultados de las puntuaciones por criterio, subcriterio y la relación con el CMI están descritos en la Tabla 2.

Tabla 2 - Comparación del BPEP con el CMI.

\begin{tabular}{|c|c|c|c|}
\hline CRITERIOS & $\mathbf{P}$. & SUBCRITERIOS & CMI \\
\hline \multirow{2}{*}{$\begin{array}{l}\text { 1. LIDERAZGO } \\
120 \text { PUNTOS }\end{array}$} & 70 & 1a. Liderazgo sénior & $\mathrm{V}$ \\
\hline & 50 & 1b. Gobernanza y responsabilidad social & $\mathrm{V}$ \\
\hline \multirow{2}{*}{$\begin{array}{l}\text { 2. PLANTEAMIENTO } \\
\text { ESTRATÉGICO } \\
85 \text { PUNTOS }\end{array}$} & 40 & 2a. Desarrollo de estrategias & $\mathrm{V}$ \\
\hline & 45 & 2b. Implementación de estrategias & $\mathrm{V}$ \\
\hline \multirow{2}{*}{$\begin{array}{l}\text { 3. ENFOQUE EN LOS } \\
\text { CLIENTES } \\
\text { 85 PUNTOS }\end{array}$} & 45 & 3a. Voz del cliente & $\mathrm{C}$ \\
\hline & 40 & 3b. Compromiso con el cliente & $\mathrm{C}$ \\
\hline \multirow{2}{*}{$\begin{array}{l}\text { 4. MEDICIÓN, } \\
\text { ANÁLISIS Y GESTIÓN } \\
\text { DEL CONOCIMIENTO } \\
90 \text { PUNTOS }\end{array}$} & 45 & 4a. Medición, análisis y mejora del desempeño organizacional & $\mathrm{F}$ \\
\hline & 45 & 4b. Gestión de la información y del conocimiento & A \\
\hline \multirow{2}{*}{$\begin{array}{l}\text { 5. ENFOQUE EN LAS } \\
\text { PERSONAS } \\
\text { 85 PUNTOS } \\
\end{array}$} & 40 & 5a. Ambiente laboral & A \\
\hline & 45 & 5b. Compromiso laboral & A \\
\hline \multirow{2}{*}{$\begin{array}{l}\text { 6. ENFOQUE } \\
\text { OPERACIONAL } \\
85 \text { PUNTOS }\end{array}$} & 45 & 6a. Sistemas de trabajo & $\mathrm{P}$ \\
\hline & 40 & 6b. Procesos de trabajo & $\mathrm{P}$ \\
\hline \multirow{5}{*}{$\begin{array}{l}\text { 7. RESULTADOS } \\
450 \text { PUNTOS }\end{array}$} & 120 & 7a. Resultados de productos y servicios & $\mathrm{P}$ \\
\hline & 90 & 7b. Resultados de enfoque al cliente & $\mathrm{C}$ \\
\hline & 80 & 7c. Resultados de enfoque en las personas & A \\
\hline & 80 & 7d. Resultados de liderazgo y gobernanza & $\mathrm{V}$ \\
\hline & 80 & 7e. Resultados financieros y del mercado & $\mathrm{F}$ \\
\hline TOTAL & 1.000 & & \\
\hline
\end{tabular}

Notas: $\mathrm{V}=$ Visión y estrategia; $\mathrm{F}=$ Financiera $\mathrm{C}=\mathrm{Cliente} ; \mathrm{P}=$ Procesos; $\mathrm{A}=$ Aprendizaje y Crecimiento. Fuente: Elaboración propia. 
El Uso del Cuadro de Mando Integral como Instrumento de Medición para Comparar los Modelos de Excelencia en Gestión

Es posible observar que en el BPEP la parte central del CMI (visión y estrategia) es la que tiene la cantidad más grande de subcriterios relacionados (cinco), seguido de la perspectiva de aprendizaje y crecimiento (cuatro), clientes y procesos (tres) y por la perspectiva financiera con dos.

Sin embargo, cuando se mira la cantidad de puntos y la relación con el CMI, se percibe que la visión y estrategia es la que tiene más subcriterios y también más puntos, con 285 . La perspectiva de aprendizaje y crecimiento está en la segunda posición con 210 puntos, casi la misma puntuación de la perspectiva de procesos, que ha obtenido 205 puntos. Las dos perspectivas con menor puntuación fueron la de clientes, con 175 y la financiera con 125 puntos. El tercero modelo de excelencia en gestión evaluado fue el MEG, que contiene ocho criterios y 23 subcriterios.

La Tabla 3 presenta los criterios, puntuaciones, subcriterios y las relaciones con el CMI. En el MEG la perspectiva más relevante es la de clientes con 320 puntos. Un segundo grupo está formado por las perspectivas de aprendizaje y de procesos internos, ya que tuvieron una puntuación superior a 200 puntos, distanciando las del tercer grupo, formado por la perspectiva financiera y por la visión y estrategias con 130 y 60 puntos, respectivamente.

Tabla 3 - Comparación del MEG con el CMI.

\begin{tabular}{|c|c|c|c|}
\hline CRITERIOS & P. & SUBCRITERIOS - MEG & CMI \\
\hline \multirow{3}{*}{$\begin{array}{l}\text { 1. LIDERAZGO } \\
110 \text { PUNTOS }\end{array}$} & 40 & $\begin{array}{l}\text { 1a. Los líderes implementan procesos de gestión que contribuyen al } \\
\text { compromiso de la organización con la excelencia, con la sostenibilidad y con } \\
\text { la transparencia. }\end{array}$ & $\mathrm{C}$ \\
\hline & 40 & $\begin{array}{l}\text { 1b. Los líderes implementan procesos de gestión que contribuyen a la } \\
\text { participación de los trabajadores y de las otras partes interesadas en el éxito de } \\
\text { las estrategias y del excelencia. }\end{array}$ & A \\
\hline & 30 & $\begin{array}{l}\text { 1c. Los líderes implementan procesos de gestión que contribuyen a evaluar el } \\
\text { desempeño operacional y estratégico en relación con los objetivos. }\end{array}$ & $\mathrm{P}$ \\
\hline \multirow{2}{*}{$\begin{array}{l}\text { 2. ESTRATEGIAS } \\
\text { Y PLANES } \\
\text { 60 PUNTOS }\end{array}$} & 30 & $\begin{array}{l}\text { 2a. Los procesos de gestión contribuyen a la generación de estrategias } \\
\text { coherentes y consistentes. }\end{array}$ & $\mathrm{V}$ \\
\hline & 30 & $\begin{array}{l}\text { 2b. Los procesos de gestión garantizan el despliegue, la creación y la } \\
\text { actualización de las estrategias de la organización. }\end{array}$ & $\mathrm{V}$ \\
\hline \multirow{2}{*}{$\begin{array}{l}\text { 3. CLIENTES } \\
60 \text { PUNTOS }\end{array}$} & 30 & $\begin{array}{l}\text { 3a. Los procesos de gestión contribuyen a satisfacer las necesidades y } \\
\text { expectativas de los clientes, a hacer de los productos y de las marcas conocidos } \\
\text { y a tornar la imagen favorable. }\end{array}$ & $\mathrm{C}$ \\
\hline & 30 & $\begin{array}{l}\text { 3b. Los procesos de gestión contribuyen a la satisfacción del cliente y } \\
\text { a lealtad a los productos y marcas. }\end{array}$ & $\mathrm{C}$ \\
\hline \multirow{2}{*}{$\begin{array}{l}\text { 4. SOCIEDAD } \\
60 \text { PUNTOS }\end{array}$} & 30 & $\begin{array}{l}\text { 4a. Los procesos de gestión contribuyen a la generación de productos, procesos } \\
\text { e instalaciones seguras para los usuarios y para el medio ambiente, } \\
\text { promoviendo el desarrollo sostenible. }\end{array}$ & C \\
\hline & 30 & $\begin{array}{l}4 \text { b. Los procesos de gestión estimulan el desarrollo social y promueven una } \\
\text { imagen favorable de la organización en la sociedad. }\end{array}$ & $\mathrm{C}$ \\
\hline \multirow{2}{*}{$\begin{array}{l}\text { IFORMACIÓN Y } \\
\text { CONOCIMIENTO } \\
\quad \text { 60 PUNTOS }\end{array}$} & 30 & $\begin{array}{l}\text { 5a. Los procesos de gestión contribuyen a la disposición sistemática de } \\
\text { información precisa y actualizada y segura para los usuarios, con el apoyo de } \\
\text { las tecnologías de la información. }\end{array}$ & A \\
\hline & 30 & $\begin{array}{l}\text { 5b. Los procesos de gestión contribuyen a aumentar la ventaja competitiva a } \\
\text { través del desarrollo y de la protección de los activos intangibles. }\end{array}$ & A \\
\hline \multirow{3}{*}{$\begin{array}{l}\text { 6. PERSONAS } \\
\text { 90 PUNTOS }\end{array}$} & 30 & $\begin{array}{l}\text { 6a. Los procesos de gestión contribuyen al alto desempeño de las personas y de } \\
\text { los equipos. }\end{array}$ & A \\
\hline & 30 & $\begin{array}{l}\text { 6b. Los procesos de gestión contribuyen a la formación y al desarrollo de los } \\
\text { miembros de la fuerza de trabajo. }\end{array}$ & A \\
\hline & 30 & $\begin{array}{l}\text { 6c. Los procesos de gestión contribuyen a la creación de un ambiente seguro y } \\
\text { saludable y a alcanzar el bienestar, la satisfacción y el compromiso de las } \\
\text { personas. }\end{array}$ & A \\
\hline \multirow{2}{*}{$\begin{array}{l}\text { 7. PROCESOS } \\
110 \text { PUNTOS }\end{array}$} & 50 & $\begin{array}{l}\text { 7a. Los procesos de gestión contribuyen a las actividades operacionales y } \\
\text { aseguran la generación de productos excelentes para los clientes. }\end{array}$ & $\mathrm{P}$ \\
\hline & 30 & $\begin{array}{l}\text { 7b. Los procesos de gestión contribuyen al desarrollo, a la mejora de la cadena } \\
\text { de suministro y al compromiso de los proveedores y socios a la excelencia. }\end{array}$ & $\mathrm{P}$ \\
\hline
\end{tabular}


El Uso del Cuadro de Mando Integral como Instrumento de Medición para Comparar los Modelos de Excelencia en Gestión

\begin{tabular}{|c|c|c|c|}
\hline & 30 & $\begin{array}{l}\text { 7c. Los procesos de gestión contribuyen a la sostenibilidad económica y } \\
\text { financiera de la organización. }\end{array}$ & $\mathrm{F}$ \\
\hline \multirow{6}{*}{$\begin{array}{l}\text { 8. RESULTADOS } \\
450 \text { PUNTOS }\end{array}$} & 100 & $\begin{array}{l}\text { 8a. El análisis económico de los resultados financieros de la organización, } \\
\text { incluidos los relativos a la estructura, la liquidez, la actividad y la rentabilidad. }\end{array}$ & $\mathrm{F}$ \\
\hline & 100 & $\begin{array}{l}\text { 8b. El análisis de los resultados relativos a los clientes y a los mercados, } \\
\text { incluidos los relacionados con la imagen de la organización. }\end{array}$ & $\mathrm{C}$ \\
\hline & 60 & $\begin{array}{l}\text { 8c. El análisis de los resultados relativos a la sociedad, incluidos los relativos a } \\
\text { la responsabilidad ambiental y al desarrollo social. }\end{array}$ & $\mathrm{C}$ \\
\hline & 60 & $\begin{array}{l}\text { 8d. El análisis de los resultados relativos a las personas, incluidos los } \\
\text { relacionados a los sistemas de trabajo, a la formación, al desarrollo, a la } \\
\text { calidad de vida y a la dirección de personas. }\end{array}$ & A \\
\hline & 100 & $\begin{array}{l}\text { 8e. El análisis de los resultados relacionados a los productos y a los principales } \\
\text { procesos de negocio y de apoyo, así como a los otros procesos de gestión de } \\
\text { resultados. }\end{array}$ & $\mathrm{P}$ \\
\hline & 30 & $\begin{array}{l}\text { 8f. El análisis de los resultados relativos a los productos recibidos y a la } \\
\text { gestión de los proveedores. }\end{array}$ & $\mathrm{P}$ \\
\hline TOTAL & 1.000 & & \\
\hline
\end{tabular}

Notas: $\mathrm{V}=$ Visión y estrategia; $\mathrm{F}=$ Financiera; $\mathrm{C}=\mathrm{Cliente}$; $\mathrm{P}=$ Procesos; $\mathrm{A}=$ Aprendizaje y Crecimiento. Fuente: Elaboración propia.

Con base en los resultados obtenidos con las evaluaciones de los tres modelos, fue posible crear la Figura 6, que representa el $\mathrm{CMI}$, los subcriterios relacionados con cada perspectiva y las puntuaciones obtenidas por los modelos EFQM, BPEP y MEG.

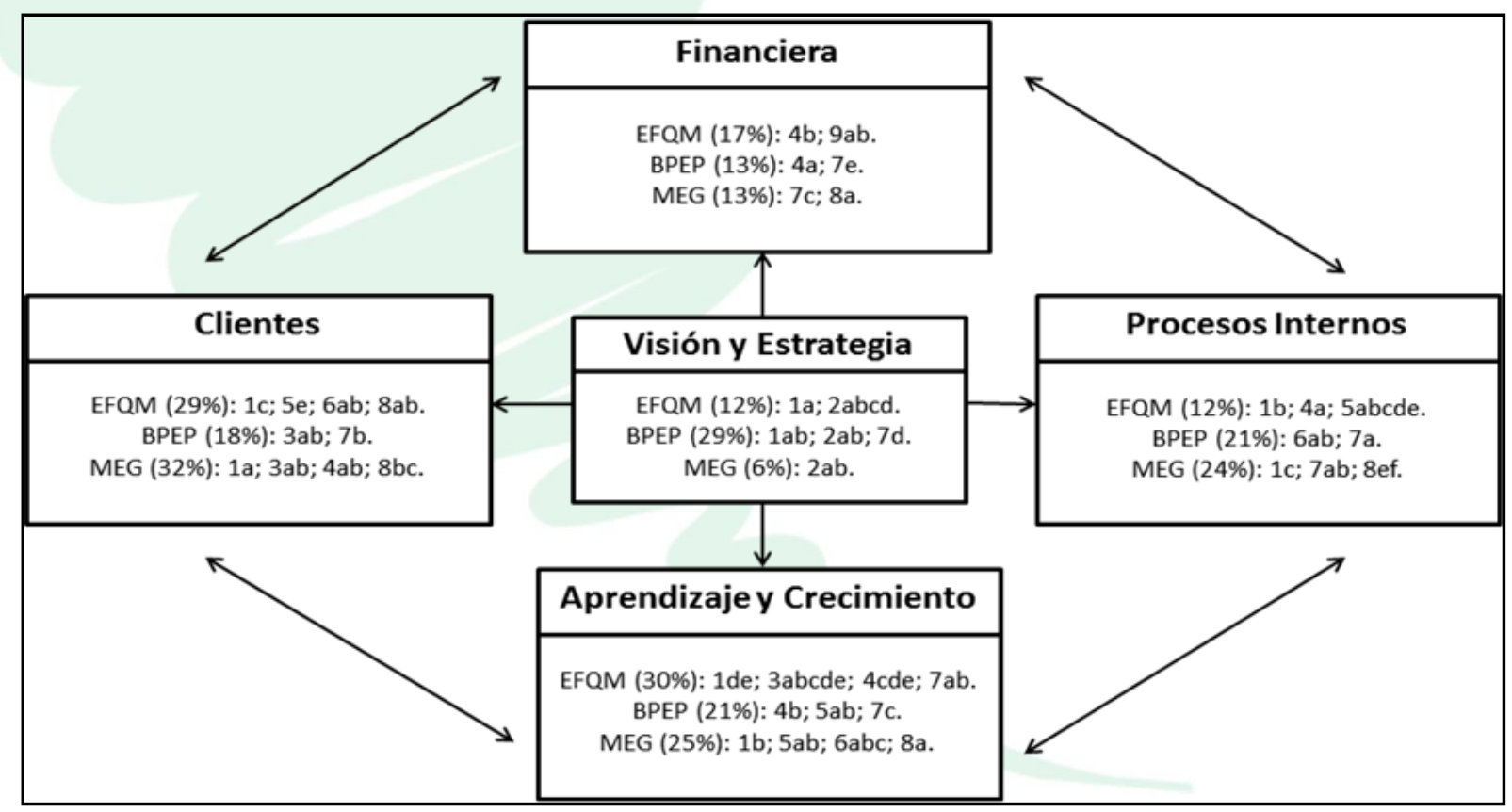

Figura 6 - Relaciones entre el CMI y los modelos de excelencia en gestión.

Fuente: Elaboración propia.

El porcentaje que se enseña al lado del nombre de cada modelo de excelencia en la Figura 6, que sigue el modelo elaborado por Kaplan y Norton (1996a) sobre los relacionamientos de las perspectivas del CMI y de la visión y estrategia, fue obtenido con la suma de los pesos de cada subcriterio presentado en las Tablas 1, 2 y 3. Con el objetivo de facilitar la comparación entre los porcentajes, fue elaborada la
Figura 7, en formato gráfico, que compara la puntuación obtenida en cada perspectiva por los modelos de excelencia en gestión. 
El Uso del Cuadro de Mando Integral como Instrumento de Medición para Comparar los Modelos de Excelencia en Gestión

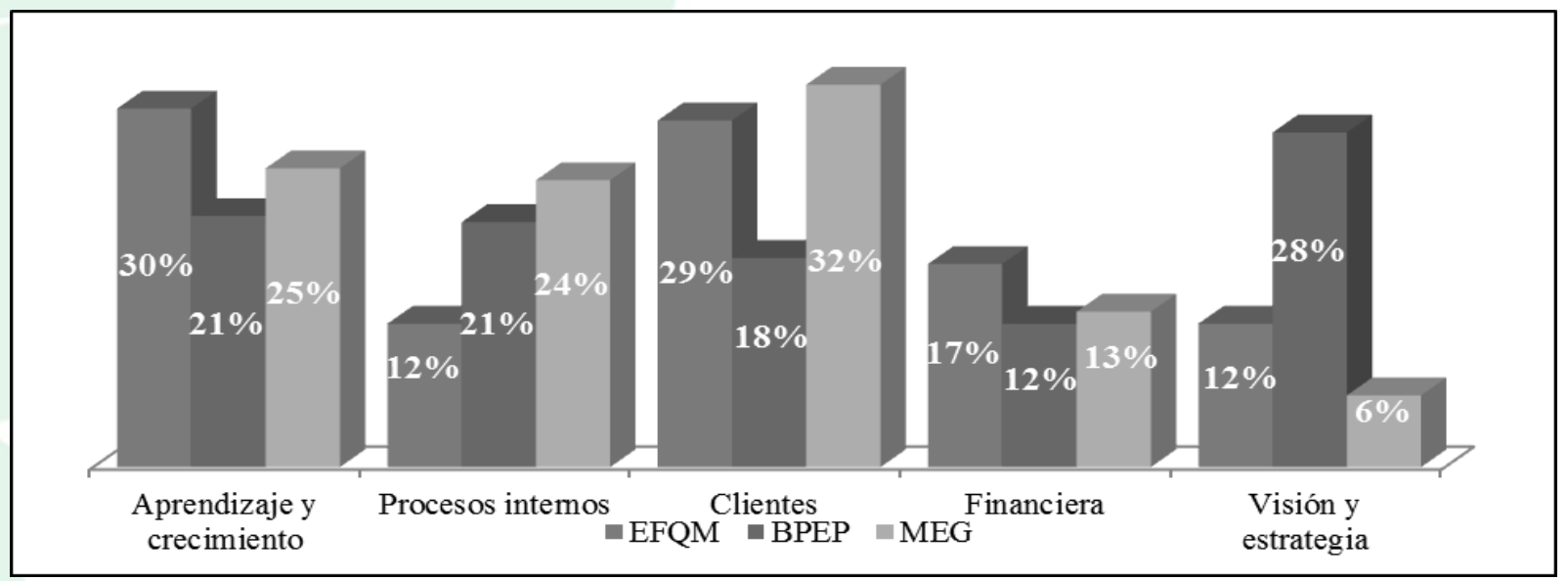

Figura 7 - Comparación entre la puntuación por perspectiva del CMI. Fuente: Elaboración propia.

Es posible observar en los resultados presentados que todos los modelos de excelencia en gestión difieren cuanto a la perspectiva más importante. Mientras para el EFQM la perspectiva de aprendizaje y crecimiento se mostró la más importante, con 30\% de los puntos, para el BPEP fue la visión y estrategia con $28 \%$ y para el MEG la perspectiva clientes, con $32 \%$. Sin embargo, percibiese que la perspectiva financiera es la única que no ha recibido más de 200 puntos (20\%) por ningún modelo.

\section{CONCLUSIONES Y RECOMENDACIONES}

El presente trabajo se ha propuesto analizar comparativamente los modelos de excelencia en gestión EFQM, BPEP y MEG bajo las perspectivas del CMI. Similitudes han sido encontradas entre los tres modelos. La primera en cuanto al objetivo que ha originado sus creaciones, segundo en cuanto a su naturaleza no prescriptiva y por ultimo también coinciden en cuanto a la puntuación máxima que una empresa puede obtener (1.000 puntos) bajo su evaluación.

Sin embargo, al reasignar los subcriterios de cada modelo de gestión, a las cinco perspectivas del CMI, se ha presentado un panorama muy diferente. Por ejemplo, la perspectiva visión y estrategia del CMI, se ha mostrado como la perspectiva más importante para el modelo americano BPEP, con peso del 28\%, mientras que para los modelos europeo EFQM (12\%) y brasileño MEG (6\%) ha sido la menos importante.

Viniendo de bajo para arriba, tenemos la perspectiva de aprendizaje y crecimiento, que ha parecido la más equilibrada entre los tres modelos evaluados (EFQM el 30\%, BPEP el $21 \%$ y MEG el $25 \%$ ). Así que hay unanimidad en los tres modelos americano, europeo y brasileño en el hecho de dar mucha importancia al definir los objetivos de la perspectiva aprendizaje $\boldsymbol{y}$ crecimiento para conseguir la misión y la visión de una organización.

La perspectiva de procesos internos, para el modelo europeo EFQM, posee un interés menor, ya que sólo fue posible relacionar el $12 \%$ de los subcriterios del modelo EFQM con la perspectiva procesos internos. Sin embargo, los procesos internos, también aparecen como una de las perspectivas claves para los modelos norteamericano BPEP (21\%) y brasileño MEG (24\%).

La perspectiva de clientes ha recibido la mayor puntuación de los modelos EFQM (30\%) y MEG (29\%), que parecen valorar más el enfoque en los clientes, mientras que para el BPEP (18\%), ha sido la perspectiva que ha obtenido la segunda menor puntuación.

La perspectiva de la parte de arriba del CMI, la perspectiva la financiera, define el fin último de la organización y ha presentado un cierto equilibrio entre los modelos evaluados, así como la de aprendizaje y crecimiento. Sin embargo, ha presentado valores mucho menores $(17 \%, 12 \%$ y $13 \%)$.

Es posible ver que hay una relación complementaria entre los modelos de excelencia en gestión y el cuadro de mando integral, ya que el EFQM, el BPEP y el MEG pueden utilizar el CMI para alinear los indicadores evaluados en cada subcritero, con la visión y estrategia de la empresa, priorizar y garantizar recursos para los procesos clave, aprovecharlo para poder comunicar la estrategia de forma más eficaz y también para tener un enfoque hacia los resultados financieros. Ya que todos los modelos han presentado una puntuación poco expresiva en esta perspectiva, pero es lógico que así sea. Cuando una empresa invierte en formación y busca alianzas con los mejores, mejora sus procesos. Cuando los procesos mejoran, los clientes lo perciben y como 
El Uso del Cuadro de Mando Integral como Instrumento de Medición para Comparar los Modelos de Excelencia en Gestión

consecuencia los beneficios aumentan. El CMI puede utilizar el sistema de evaluación de los modelos de excelencia en gestión (EFQM, BPEP y MEG) para alinear sus objetivos a las metas de la organización. Por fin, se sugiere que las empresas multinacionales busquen conocer los diversos modelos de excelencia en gestión y adaptarlos a sus necesidades locales, ya que podrán encontrar muchos modelos, dependiendo de la cantidad de países en los que actúen.

\section{REFERENCIAS}

Akhavan, P.; Shirazi, H.; Sabzaligol, A.; Pezeshkan, A. (2013). A Framework for Organizational Knowledge Assessment by Combining BSC and EFQM: A Case of Beasat Industry Complex, Iran. The IUP Journal of Knowledge Management, v. XI, n. 2, p. 7-18.

América Economía. (2013). Especial multilatinas. Disponible en: http://rankings.americaeconomia.com/2013/ranking _multilatinas_2013/ranking.php. Acceso en: 07 sep. 2014.

Bardin, L. (2011). Análise de conteúdo. São Paulo: Edições 70, p. 229.

Correa, H.L.; Paiva, E.L.; Primo, M.A.M. (2010). A pesquisa em Gestão de Operações no Brasil: um Breve Relato de sua Evolução. RAE Eletrônica,v. 9, n. 2.

Dror, S. (2008). The Balanced Scorecard versus quality award models as strategic frameworks. Total Quality Management, v. 19, n. 6, p. 583-593.

EFQM - European Foundation for Quality Management. (2013a). The EFQM Excellence Model. Disponible en: <http://www.efqm.org>. Acceso en: 14 jun. 2013.

EFQM - European Foundation for Quality Management. (2013b). Introducing the EFQM Excellence Model 2010. Disponible en $<$ http://www.efqm.org/en/PdfResources/EFQMMo del Presentation.pdf>. Acceso en: 18 jun. 2013.

Godoy, A.S. (1995). Introdução à pesquisa qualitativa e suas possibilidades. RAE - Revista de Administração de Empresas, v. 35, n. 2, p. 57-63.

FDC - Fundação Don Cabral. (2013). Ranking FDC das Multinacionais Brasileiras 2013: os Impactos da Política Externa na Internacionalização das Empresas Brasileiras. Disponible en <http://www.google.com.br/url?sa=t\&rct=j\&q=\&es $\underline{\mathrm{rc}=}=\mathrm{s} \&$ frm $=1 \&$ source $=$ web $\& \mathrm{~cd}=2 \&$ ved $=0 \mathrm{CCMQFj}$ AB\&url=http\%3A\%2F\%2Fwww.fdc.org.br\%2Fim prensa $\% 2$ FDocuments $\% 2$ F2013\%2Franking multi nacionais brasileiras2013.pdf\&ei=7KQMVJ7kM4b isASx2oKIBw\&usg=AFQjCNHBJO9qAnoOkWA YhtidNeXKiupBFw\&bvm=bv.74649129,d.cWc>. Acceso en: 06 sep. 2014.

FNQ - Fundação Nacional da Qualidade. (2009). Critérios de Excelência, São Paulo, Fundação Nacional da Qualidade.

Kaplan, R.S.; Norton, D.P. (1992). The Balanced Scorecard: Measures that Drive Performance. Harward Business Review, p. 71-79.

Kaplan, R.S.; Norton, D.P. (1996a). Using the Balanced Scorecard as a Strategic Management System, Harward Business Review, p. 75-85.

Kaplan, R.S.; Norton, D P. (1996b). Linking the Balanced Scorecard to Strategy, California Management Review, v. 39, n. 1, p. 53-79.

Marconi, M.D.A.; Lakatos, E.M. (2009). Metodologia do trabalho científico: procedimentos básicos, pesquisa bibliográfica, projeto e relatório, publicações e trabalhos científicos. 7. ed. São Paulo: Atlas.

Miguel, P.A.C. (2001). Comparing the Brazilian national quality award with some of the major prizes, The TQM Magazine, v. 13, n. 4, p. 260-272.

NIST - National Institute of Standards and Technology. (2013). Criteria for Performance Excellence 2011$2012 . \quad$ Disponible en <http://www.nist.gov/baldrige/publications/archive/ 2011_2012_business_nonprofit_criteria.cfm>. Acceso en: jun. 2013.

Richardson, R.J. (2009). Pesquisa social: métodos e técnicas. São Paulo: Atlas.

Rovai, R.L.; Campanário, M.A.; Costa, T.R. (2011). Multinacionais Brasileiras - Evolução e Perspectivas Teóricas: Caracterização de uma Tipologia. Revista Inteligência Competitiva, v. 1, n. 2, p. 205-217.

Pastor A.C.; Pastor J.P.; Ascoz, J. M. C.; Pérez, M. A. R. y Elola, L. N. (2013). Comparison of Models Evaluating Business Excellence, Tourism \& Management Studies, v. 4, special issue, p. 10581072. 
El Uso del Cuadro de Mando Integral como Instrumento de Medición para Comparar los Modelos de Excelencia en Gestión

Pastor J.; Elola, L.N. y Pastor A. C. (2008). The application of neural networks in the study of the influence of temporality on strategy map indicators in a Spanish hospital, Total Quality Management, v. 19, n. 6, p. 643-659.

Vasconcellos, A.L.C.; Lucas, S. F. (2012). Análise Crítica da Metodologia BSC na Visão da
Excelência Em Gestão. VIII Congresso Nacional de Excelência em Gestão.

Vilas Boas, G.A.R.; Costa, H. G. (2011). Análise Comparativa de Prêmios de Excelência em Gestão. VII Congresso Nacional de Excelência em Gestão. 